\title{
Theoretical and experimental study of the bleaching of a dye in a film-polymerization process
}

\author{
Luis Carretero, Salvador Blaya, Ricardo Mallavia, Roque Fernando Madrigal, \\ Augusto Beléndez, and Antonio Fimia
}

\begin{abstract}
The quantum efficiency and the molar-absorption coefficients of different phenothiazine dyes are obtained by means of fitting the experimental data of transmittance as a function of time. An analytical expression for the intensity transmitted in a photopolymerizable holographic material is obtained, and good agreement between theory and experience is also achieved. The analysis of these parameters is of fundamental quantities in the photochemical characterization of holographic recording materials. (C) 1998 Optical Society of America

OCIS code: $\quad 090.2900$.
\end{abstract}

Different studies of the photoprocesses of dye molecules trapped in transparent solid matrices have been carried out. ${ }^{1-4}$ The dye's quantum yield and the molar-absorption coefficient are two basic parameters that will determine the photochemical behavior of the material into which the dye has been introduced. The quantum yield of a photochemical reaction is the number of product moles that form divided by the number of photons absorbed.

Usually the study of the molar-absorptivity coefficient is carried out in a static way by use of experimental data obtained with a spectrophotometer, particularly when studying the instantaneous absorption of a sample for a fixed wavelength. On the other hand, the generation radical's quantum yield has been measured (in solution) by laser-flash photolysis. ${ }^{5}$ In this Note we apply a dynamic study of the temporal evolution of transmittance by using a nonlinear fit of these curves to obtain the quantum yield of the generated radicals and the molar-

L. Carretero is with the Laboratorio de Optica, Interuniversitario de Optica, and A. Beléndez is with the Departamento de Física, Ingenieria de Sistemas y Teoria de la Seńal, Universidad de Alicante, Apdo. No. 99, E-03080 Alicante, Spain. S. Blaya, R. Mallavia, R. F. Madrigal, and A. Fimia are with the Departamento de Ciencias Experimentales y Tecnología, Universidad Miguel Hernández, Avenido Ferrocarril s/n, Ed Galia, 03202 Elche (Alicante), Spain.

Received 14 August 1997; revised manuscript received 26 March 1998.

0003-6935/98/204496-04\$15.00/0

(C) 1998 Optical Society of America absorption coefficient in a photopolymer system used as a recording material.

When organic dye molecules are illuminated in the absorption band excited forms are produced, and these excited forms can be converted into radical molecules or can return to the nonexcited state by quenching processes. Reactions such as combinations and oxidations or reductions of these radicals produce other components that normally do not absorb in the dye's band (i.e., the process of bleaching).

Consider a dry polymeric film of thickness $d$ containing a dye $A$ that reacts photochemically when it is illuminated with a monochromatic laser beam of intensity $I_{0}$ (in einsteins per centimeter squared times seconds). The initial concentration of $A$ is $A_{0} \mathrm{~mol} /$ $\mathrm{cm}^{3}$. In the film, the concentration of $A$ at a time $t$ is $A(t)$, and the absorbed intensity at the same time is $I a(t)$. The local rate equation for the dye in a photopolymerizable mixture (the mechanism of the reaction is described in Fig. 1) will be

$$
\frac{\mathrm{d} A(t)}{\mathrm{d} t}=-\frac{\phi \operatorname{Ia}(t)}{d}
$$

where $\phi$ is the quantum yield.

With reference to the reaction mechanism shown in Fig. 1, it is important to say that the bleaching reaction of the dye is produced by the absorption of one photon by a photoinitiator with conversion to its triplet state. Then a redox reaction takes place between the excited dye molecule and the amine, generating the semireduced dye radical. A second electron transfer between the amine and the radical and a 


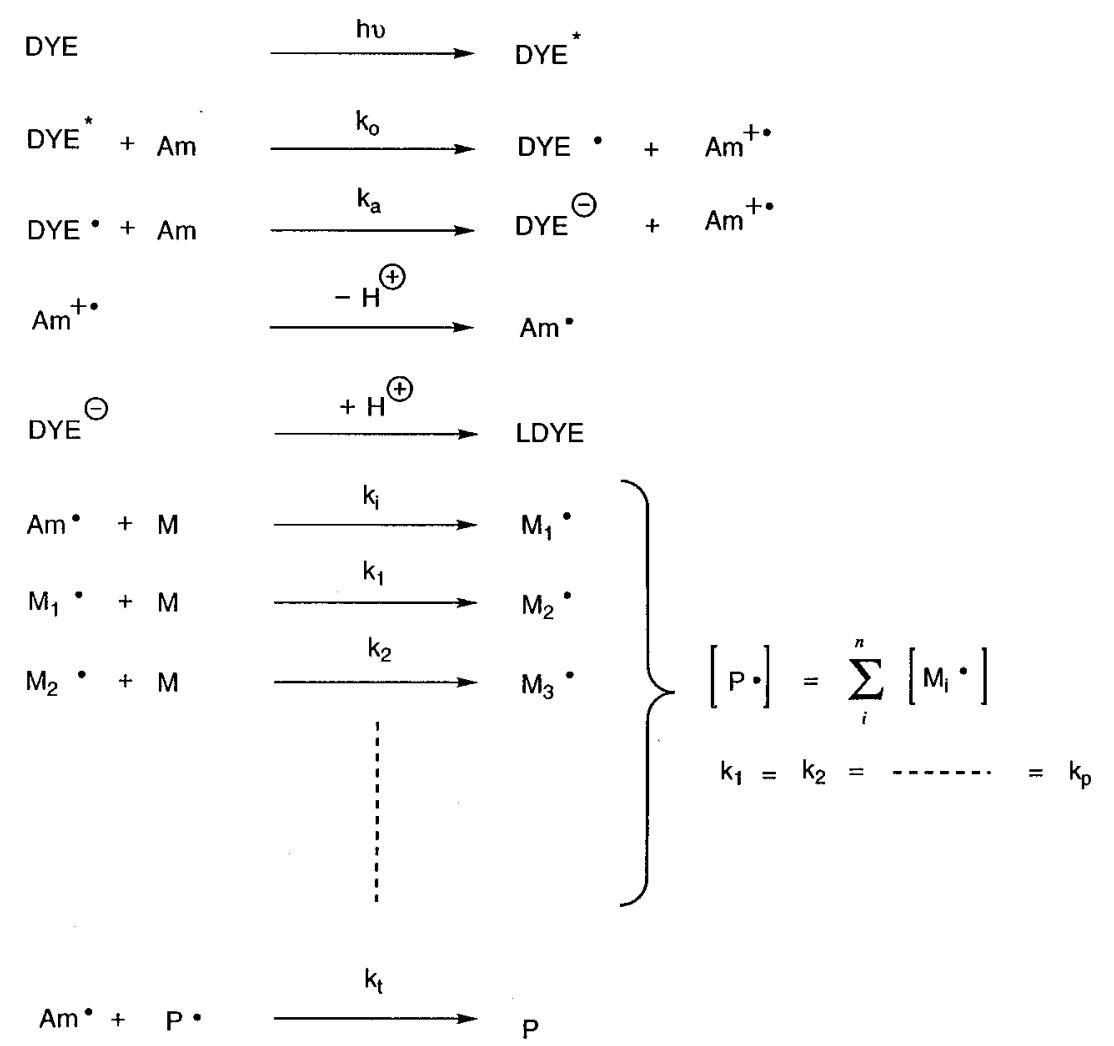

Fig. 1. Scheme of the proposed mechanism of photopolymerization: DYE, the photoinitiator; Am, amine; P, polymer; M, monomer; DYE, photoinitiator; $\mathrm{DYE}^{*}$, excited photoinitiator; $\mathrm{DYE}^{*}$, radical photoinitiator derivative; $\mathrm{DYE}^{\ominus}$, anion derivative of the photoinitiator; $\mathrm{LDYE}$, leuco-dye; $\mathrm{Am}$, amine; $\mathrm{Am}^{+}$, cation radical; $\mathrm{AM}^{\bullet}$, radial derived from amine; $\mathrm{M}$, monomer; $\mathrm{P}$, polymer; $\mathrm{M}_{\mathrm{i}}$, polymer radical with i subunits; $\mathrm{h} v$, energy of an incident photon; K, reaction constant.

protonation process give rise to a leuco-form of the dye. We assume that the quantum yield of the dye destruction is equal to the quantum yield of the radical generation.

The absorbed intensity is given by the LambertBeer equation, ${ }^{5}$ which can be expressed as

$$
I a(t)=I_{0}\{1-\exp [-\epsilon A(t) d]\}
$$

where $\epsilon$ is the molar-absorption coefficient.

Relating Eqs. (2) and (1) allows the expression for the absorbed intensity to be expressed by

$$
A(t)=(\epsilon d)^{-1} \ln \left\{1+\left[\exp \left(\epsilon d A_{0}\right)-1\right] \exp \left(-\epsilon \phi I_{0} t\right)\right\}
$$

Introducing Eq. (3) into Eq. (2) yields the time evolution of the absorbed intensity:

$$
I a(t)=\frac{I_{0}\left[\exp \left(\epsilon d A_{0}\right)-1\right] \exp \left(-\epsilon \phi I_{0} t\right)}{1+\left[\exp \left(\epsilon d A_{0}\right)-1\right] \exp \left(-\epsilon \phi I_{0} t\right)} .
$$

When light falls upon the medium, a portion of the incident intensity $I a(t)$ is absorbed, and the remain$\operatorname{der} I_{T}(t)$ is transmitted. Then we have

$$
I_{0}=I_{a}(t)+I_{T}(t) .
$$

Then the transmittance $T(t)$, defined as $I_{T}(t) / I_{0}$, can be expressed as a function of time in the form of

$$
T(t)=\frac{T s f}{1+\left[\exp \left(\epsilon d A_{0}\right)-1\right] \exp \left(-\epsilon \phi I_{0} t\right)},
$$

where $T s f=(T s) T f$ is a parameter that takes into account the scattered $T s$ and the reflected $T f$ light, as obtained from Fresnel formulae.

The system used in these experiments was composed of acrylamide and triethanolamine as the coinitiator, photoinitiated with three differents dyes [methylene blue (MB), thionine (Th), and Azure-C (Ac)]. Components are supported by a film of poly(vinylalcohol) (PVA) with a molecular weight of $\approx 25,000$. The photosensitive aqueous solution was prepared when more than $50 \mathrm{ml}$ of PVA (10\% by weight), the necessary volume of dye solution of a concentration of $0.2 \mathrm{mM}$, and $8 \mathrm{ml}$ of $2.5-\mathrm{M}$ acrylamide and 1.5-M triethanolamine were combined. We prepared the film by coating a photosensitive solution over a $20 \mathrm{~cm} \times 40 \mathrm{~cm}$ glass (BK7) with a thin-layer chromatography coater and allowing it to dry for $20 \mathrm{~h}$ under normal conditions (65\% relative humidity and $20^{\circ} \mathrm{C}$ ). The resulting thickness of the film was approximately $30 \mu \mathrm{m}$.

The experimental setup for measuring the transmitted intensity is shown in Fig. 2. A 35-mW He-Ne laser with a wavelength of $633 \mathrm{~nm}$ provided a linear 


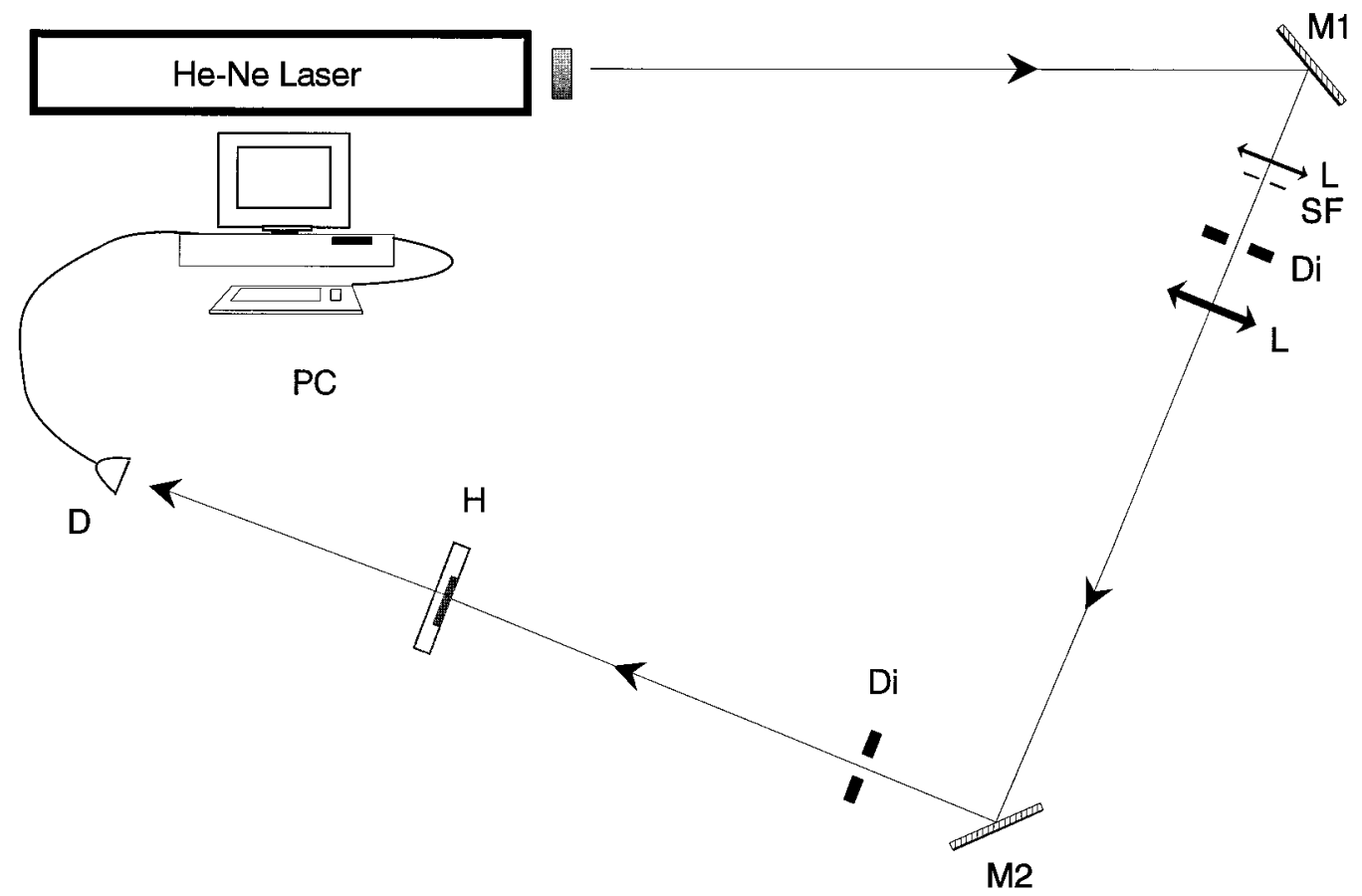

Fig. 2. Experimental setup used for the measurement of the transmitted intensity. D, detector; Di, diapragm; H, plate; M, mirror; L, lens; SF, spatial filter.
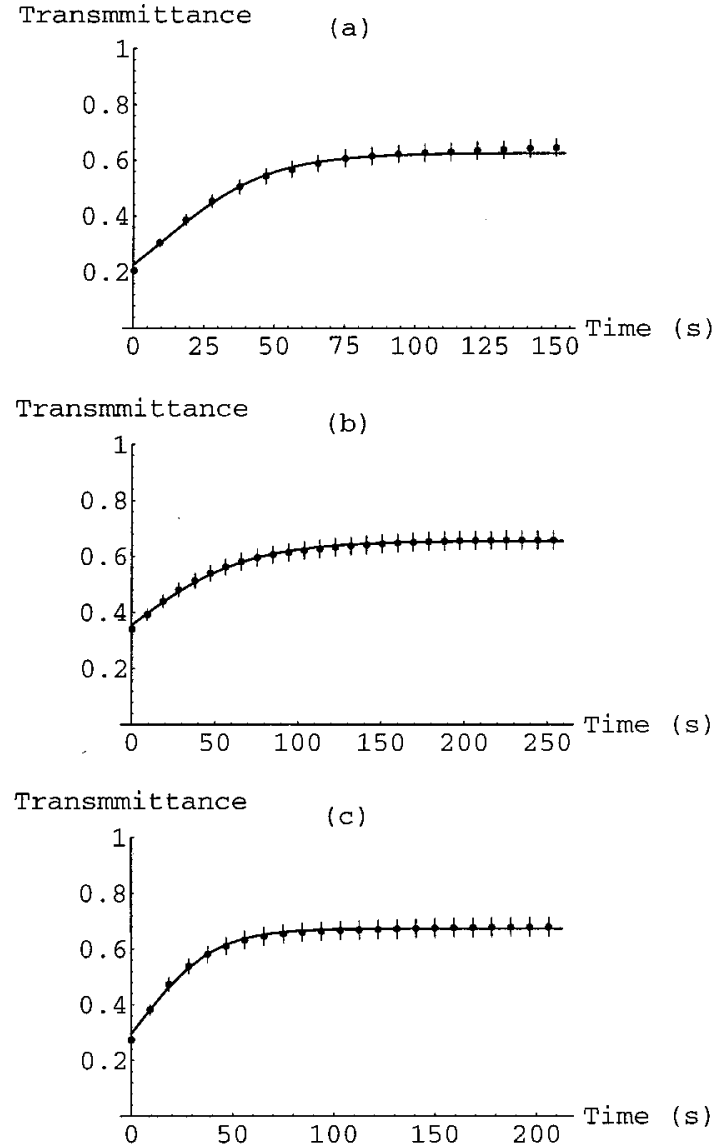

Fig. 3. Transmittance curves as a function of time for the three dyes studied: (a) MB. (b) Th. (c) Ac. polarized plane wave that is incident normal to the sample after it has been expanded to obtain a spot diameter of $1 \mathrm{~cm}$. A program that uses the $\mathrm{C}++$ compiler was written to monitor the evolution of the transmission through the sample as a function of time by use of a personal computer. Applying a nonlinear fitting procedure based on the LevenbergMarquardt algorithms ${ }^{6}$ provides values of the quantum yield and the molar-absorption coefficient.

Experiments were carried out for Th, Ac, and MB at a fixed incident intensity $\left(4.1 \mathrm{~mW} / \mathrm{cm}^{2}\right)$ and a concentration of dye equal to $0.2 \mathrm{mM}$ in all experiments. The measurements obtained and the fitted curves are shown in Fig. 3. As can be seen from this figure, the agreement between theory and experiment is good. These curves were fitted by use of Eq. (6), and the results for the quantum yield and the absorption coefficient ${ }^{7}$ are shown in Table 1.

Table 1 shows that the quantum yield of Ac is larger than the quantum yields of Th and MB. These results agree with the fact that the maximum absorption of Ac is closer to $633 \mathrm{~nm}$ than is that of MB and Th, as can be seen in Fig. 4, where the absorption curves for different wavelengths as obtained with a spectrophotometer are represented. The parameter

Table 1. Quantum Yield $\phi$, Absorption Coefficient $\epsilon$, and Tsf Parameter for $\lambda=633 \mathrm{~nm}$ Obtained by the Fitting of the Transmittance Curves

\begin{tabular}{lccc}
\hline Dye & $\epsilon\left(\mathrm{cm}^{2} / \mathrm{mol}\right)$ & $\phi(\mathrm{mol} /$ einstein $)$ & $T s f$ \\
\hline MB & $7.29 \times 10^{7}$ & 0.032 & 0.655 \\
Ac & $5.88 \times 10^{7}$ & 0.050 & 0.675 \\
Th & $4.88 \times 10^{7}$ & 0.027 & 0.669 \\
\hline
\end{tabular}




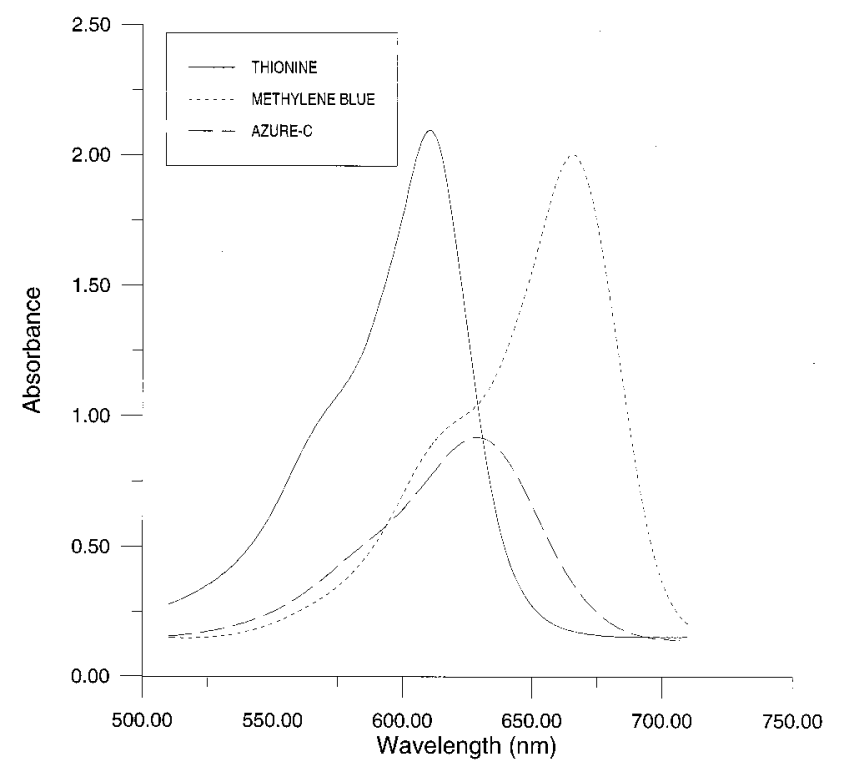

Fig. 4. Absorption spectra for the three dyes studied in a film of PVA.

$T s f$ that takes into account the light not transmitted owing to scattering and reflection is similar for the three dyes, which is an expected result because scattering and reflections are produced by the PVA solid matrix.

In conclusion, a nonlinear fit of the experimental measurements of the transmittance as a function of time by use of an analytical expression for transmittance obtained with the theoretical model has pro- vided us with the quantum yield and the absorptionmolar coefficient for three different phenothiazine dyes with their absorption bands in the region of 633 $\mathrm{nm}$. The knowledge of these parameters is very important to the optimization process of holographic recording materials because the sensitivity and the time response to light of the material are dependent on them.

This study was supported by the Direcció General d'Ensenyaments Universitaris i Investigació de la Generalitat Valenciana, Spain (Project GV-C-CN-06064-96).

\section{References}

1. E. L. Simmons, "The photochemistry of solid layers. Reaction rates," J. Phys. Chem. 75, 588-590 (1971).

2. C. Carre and D. J. Lougnot, "A photochemical study of the methylene blue/acrylamide system in view of its use for holographic recording under red illumination," J. Chimie Phys. 85, 485-490 (1988).

3. N. Capolla and R. Lessard, "Real-time bleaching of methylene blue or thionine sensitized gelatin," Appl. Opt. 30, 1196-1200 (1991).

4. A. Dubois, M. Canva, A. Brun, F. Chaput, and J. Boilot, "Photostability of dye molecules trapped in solid matrices," Appl. Opt. 35, 3193-3199 (1996).

5. N. J. Turro, Modern Molecular Photochemistry (University Science, Mill Valley, Calif., 1991), p. 103.

6. K. Lavenberg, "A method for the solution of certain problems of least squares," Quart. Appl. Math. 2, 164-168 (1944); D. Marquardt, "An algorithm for least-squares estimation of nonlinear parameters," SIAM J. Appl. Math. 11, 431-441 (1963).

7. L. Michaelis and S. Granick, "Metachromasy of basic dyestuffs," J. Am. Chem. Soc. 67, 1212-1219 (1945). 